\title{
Mobility and Network Management in Heterogeneous Networks
}

\author{
Kostas Pentikousis • Ramon Agüero • \\ Symeon Papavassiliou
}

Published online: 8 June 2011

(C) Springer Science+Business Media, LLC 2011

\section{Introduction}

Multiaccess and resource management, mobility management, and network management have emerged as core topics in the design, deployment and operation of current and future networks. The research activity in these areas has grown tremendously over the past decade, addressing the pressing need for ubiquitous and cost-efficient mobile broadband. At the same time, new possibilities and communications paradigms have emerged, bringing about the appearance of new challenges, which had not been considered so far. This special issue comprises peerreviewed articles that provide answers to problems ranging from optimum access selection in overlapping mobile and wireless networks to mobile network virtualization to selfmanaged network architectures with QoS guarantees.

The advent and rapid proliferation of broadband cellular networks, on one hand, and wide deployment of Wi-Fi

\author{
K. Pentikousis $(\bowtie)$ \\ Huawei Technologies \\ European Research Centre, \\ Carnotstrasse 4, \\ 10587 Berlin, Germany \\ e-mail: k.pentikousis@huawei.com \\ R. Agüero \\ Department of Communications Engineering, \\ University of Cantabria, \\ Plaza de la Ciencia s/n, \\ 39005 Santander, Spain \\ e-mail: ramon@tlmat.unican.es \\ S. Papavassiliou \\ School of Electrical and Computer Engineering, \\ National Technical University of Athens, \\ 9 Iroon Polytechniou str., \\ Athens 15780, Greece \\ e-mail: papavass@mail.ntua.gr
}

networks, on the other, made wireless Internet access commonplace over the last decade. Today most users access the Internet using a wirelessly connected device and this majority is set only in increase over the coming years. It goes without saying that delays in $3 \mathrm{G}$ network deployments in the early 2000s gave impetus to efforts for even wider deployment of Wi-Fi hot-spots, particularly in urban environments. To many, this was meant to be only a short-term solution. But practice showed that cellular networks were not going to be able to handle on their own the ever-increasing traffic needs. In other words, it became evident that operators and users would have to deal with heterogeneous network access technologies for a considerable period of time. Thus, considerable effort was put into, for instance, alleviating the shortcomings in upper-layer protocol performance over wireless links, mobility and resource management, (multi) access selection, and resource abstraction and virtualization. In short, as the performance of standard upper layer-protocols was far from optimum over wired-cum-wireless networks, the goal was set to adopt a holistic view of the complete system, and consider not only the characteristics of the underlying technologies, but also the particular requirements of on-going services, the policies established by the user and the operator, and so on. This special issue includes articles that address research questions in this vibrant research area, namely mobile and multiaccess networks, resource management and virtualization (see Section 2 below).

This line of research was soon followed by Standards Developing Organizations (SDOs) such as 3GPP, IEEE and IETF. For example, the IEEE 802.21 working group specified the Media Independent Handover (MIH) Framework as a proposal to support mobility between heterogeneous access networks, while 3GPP developed the Interworking-Wireless LAN (I-WLAN) architecture and the Access Network Discovery and Selection Function (ANDSF). The IETF work on, for example, Performance Implications of Link Character- 
istics (PILC) and Mobility for IP: Performance, Signaling and Handoff Optimization (MIPSHOP) also relates to these efforts. Nevertheless, and in spite of significant advances in the research area of mobility and network management for heterogeneous access networks, so far, no fully working solution has come into the market matching the original technology visions. In this issue, we include an article that presents a realistic mobility management implementation for Android devices based on IEEE management standards and enabling technologies which we expect will have real-world impact on tomorrow's devices.

More recently, large initiatives funded by the European Union Framework Program 7 (including FIRE-Future Internet Research and Experimentation) and the US National Science Foundation (FIND-Future Internet Design; GENI-Global Environment for Network Innovations) suggest that for making the Internet of the Future sustainable and scalable it is a requirement, rather than a desire, to develop and test in large-scale environments enhanced, flexible and robust intrinsic management approaches.

In future networks, basic services such as routing, forwarding and monitoring, as well as advanced services such as mobility, Quality of Service, and survivability need to be supported and managed, thus complicating the overall network management process. Towards this direction, autonomicity, including control loops and feedback mechanisms and processes, as well as the information/knowledge flow used to drive control loops, becomes an enabler for selfmanageability of mobile networks. Furthermore, the proliferation of autonomicity at various levels of mobile communications is expected to facilitate dynamic media adaptation to delivery platforms, either network controlled or edge controlled. This will allow the realization of context-aware networking and enhanced media delivery, and more importantly will bring the benefits and principles of flexibility, adaptation and effectiveness closer to the mobile end-user. This special issue includes papers that cover the so-called "self-* properties" of autonomic networks and introduce future Internet architectures for mobile network management.

The articles in this special issue highlight that there are still many important topics to be addressed in the current line of work. In addition, our open call for papers also identified other aspects in which the role of management procedures for wireless and mobile networks should also be considered. In this latter group, it is worth highlighting the so-called Internet of Things (IoT), which describes a worldwide network of intercommunicating devices, capable of supporting the public good and leading to economic growth and personal enrichment of life. This (re)evolution also calls for new procedures and mechanisms for managing smart objects (see Section 2 below).

We close this brief introduction to the special issue with pointers to future research in this area. Although virtualiza- tion of mobile and wireless networks has attracted a large following, its impact over management procedures is yet to be assessed and we expect this to become a growing area of research. The adoption of mesh and other non-conventional topologies is also creating opportunities for innovations, especially when combined with real-world implementations and beyond testbed deployments. From an operational perspective, multi-operator shared infrastructures, business policies and price management are also topics that worth more future work. Towards this direction cooperation between connectivity layers and service management frameworks becomes a critical aspect for future networks that requires some breakthroughs.

\section{Preface to the special issue}

The papers included in this special issue come from two sources. First, we seeded this issue with the best 3 out of 29 papers that appeared in the Proceedings of the Second ICST Conference on Mobile Networks and Management (MONAMI), which were published by Springer in the LNICST series (vol. 68, ISBN 978-3-642-21443-1). All three papers were presented at the conference held at the University of Cantabria in Santander, Spain in September 2010, and have been extended significantly before entering the normal journal peer-review process along with 27 open-call submissions. From the latter, 6 papers were selected after a thorough peerreview process and are included in this special issue. The remaining of this editorial note serves as a short introduction to each of the nine papers included in the issue, starting with those originating in MONAMI 2010.

We start this special issue with the extended version of the paper that won the Best Paper award at MONAMI 2010. The paper entitled "Optimum selection of access networks within heterogeneous wireless environments based on linear programming techniques" by Johnny Choque et al. uses optimization techniques to analyze the best performance that can be achieved over highly heterogeneous wireless access environments. The authors define a flexible utility function, which entails the use of various access selection algorithms, and show how it can evaluate the performance and suitability of different access selection mechanisms in realistic scenarios using the information available at a more local scope.

Resource virtualization and management is emerging as a vibrant area of research with a wide range of interesting questions that remain to be answered from the mobile networking perspective. Yasir Zaki et al. have won the Best Student Paper award at MONAMI 2010 for their work on LTE mobile network virtualization. Their paper "LTE mobile network virtualization exploiting multiplexing and multi-user diversity gain" is an extended version of their 
conference paper, presenting a framework which manages the virtualization of the wireless LTE substratum and assessing the benefits in terms of spectrum management.

The third paper in this special issue is also the result of article development through MONAMI 2010. Pedro A. Aranda Gutiérrez discusses traffic engineering techniques for use at the edge of the Internet in his paper entitled "A simplified Internet routing architecture". The paper starts by illustrating the hacks employed today for traffic engineering purposes and shows how they increase the Internet's core routing table unnecessarily. Then the author proposes a new architecture which aims at minimizing the impact of such traffic engineering and security artifacts on the Internet's Default Free Zone.

Privacy and identity management in mobile networks is addressed by José M. del Álamo et al. in the paper entitled "A privacy-considerate framework for identity management in mobile services". Many of the proposals which have been put forward with respect to managing available network resources assume that all context information is always available. However, much of this information may be subject to security, trust, and privacy considerations. In order to address this limitation, the paper proposes an architecture that fosters privacy by enabling users to control the use and release of their information over wireless networks.

Network monitoring and management of smart objects calls for novel approaches in order to ensure energy efficiency and system resilience. Shouwen Lai and Binoy Ravindran define "An automatic presence service for low duty-cycled mobile sensor networks" and evaluate it through analysis and simulation. The proposed presence service for multi-hop wireless sensor networks addresses three main problems, namely, neighbor discovery, message passing, and automatic network monitoring.

The special issue includes also two works within the research area of autonomic network management. First, the paper by Panagis Magdalinos et al. entitled "Enhancing a fuzzy logic inference engine through machine learning for a self-managed network" tackles one of the key network management system requirements, i.e. minimizing the need for direct user involvement in decision-making processes. The authors present a scheme based on machine learning techniques and fuzzy logic mechanisms so as to foster automatic network management. Second, Georgios Aristomenopoulos et al. introduce "An autonomic QoS-centric architecture for integrated heterogeneous wireless networks", which jointly manages resources from heterogeneous wireless access networks. The proposed architecture guarantees quality of service (QoS) through autonomic node operation. Analytical and simulation results are presented showing the potential benefits of their proposal and a path towards its realization is introduced.
We conclude this special issue with two papers that cover the research area of multihoming and multiaccess management. Bruno M. Souza et al. provide an in-depth analysis of available multi-homing solutions at all layers of the Internet protocol stack in their paper entitled "Multihoming management for future networks". This survey of existing solutions includes a critical evaluation of multihoming support in different protocols and points to future research topics in this area.

Last but certainly not least, Ricardo Silva et al. present their implementation for multiaccess mobility management for Android smartphones, based on the IEEE 802.21 Media Independent Handover Framework and Mobile IPv6. It is worth noting that their paper entitled "Enabling heterogeneous mobility in Android devices" received the highest evaluation scores from all open-call submissions after the thorough peerreview process was concluded. In effect, Silva et al. win the unofficial best paper award for this special issue. The authors introduce their practical solution and evaluate it using a testbed. The set of measurements included in the paper indicate that a system based on the work presented in this paper could one day be part of the mobility management functionality in your next Android smartphone.

Kostas Pentikousis Is a Senior Research Engineer at Huawei Technologies. He studied computer science at Aristotle University of Thessaloniki (B.Sc. 1996) and Stony Brook University (M.Sc. 2000, Ph.D. 2004). Dr. Pentikousis has published internationally more than eighty technical papers and book chapters in several areas, including network architecture and design, mobile networking and computing, transport protocols, applications and services, local and wide-area networks, energy-efficient networking, and information-centric networking.

Ramón Agüero Received a degree in Telecommunications Engineering from the University of Cantabria in 2001 and a $\mathrm{PhD}$ in 2008. He is currently an Associate Professor at the Communications Engineering Department on that university. He has participated in several collaborative research projects. His research focuses on WLAN and WPAN technologies, with special attention on performance analysis of TCP/IP protocols over them and multi-hop (mesh) networks. He is also interested in aspects concerning heterogeneous network deployments and the Wireless Sensor Networks area. He has published more than 100 technical papers in such areas.

Symeon Papavassiliou Is an Associate Professor with the School of Electrical and Computer Engineering, National Technical University of Athens. From 1995 to 1999, Dr. Papavassiliou was a senior technical staff member at AT\&T Laboratories in Middletown, New Jersey, and in August 1999 he joined the Electrical and Computer Engineering Department at the New Jersey Institute of Technology (NJIT), where he was an Associate Professor until 2004. Dr. Papavassiliou has an established record of publications in his field of expertise, with more than one hundred and seventy technical journal and conference published papers. His main research interests lie in the area of computer and communication networks with emphasis on wireless communications, ad-hoc and sensor networks, resource allocation and optimization, and performance analysis and evaluation of stochastic systems. 